MYA/00133

\title{
Calculation of the photoionization with de-excitation cross sections of He and helium-like ions
}

\author{
M. Ya. Amusia \\ Racah Institute of Physics, The Hebrew University, Jerusalem 91904, \\ Israel; A. F. Ioffe Physical-Technical Institute, St. Petersburg, 194021, Russia \\ R. Krivec \\ Department of Theoretical Physics, J. Stefan Institute, \\ P.O. Box 3000, 1001 Ljubljana, Slovenia \\ E. Z. Liverts and V. B. Mandelzweig \\ Racah Institute of Physics, The Hebrew University, Jerusalem 91904, Israel
}

\begin{abstract}
We discuss the results of the calculation of the photoionization with de-excitation of excited He and helium-like ions $\mathrm{Li}^{+}$and $\mathrm{B}^{3+}$ at high but non-relativistic photon energies $\omega$. Several lower ${ }^{1} S$ and ${ }^{3} S$ states are considered. We present and analyze the ratios $R_{d}^{+*}$ of the cross sections of photoionization with de-excitation, $\sigma_{(d)}^{+*}(\omega)$, and of the photo-ionization with excitation, $\sigma^{+*}(\omega)$. The dependence of $R_{d}^{+*}$ on the excitation of the target object and the charge of its nucleus is presented. Apart to theoretical interest, results obtained can be verified using such long living excited state as $2^{3} S$ of He.
\end{abstract}

PACS numbers: $32.80 . \mathrm{Fb}, 31.15 . \mathrm{Ja}$ 
TABLE I: Values of the ratios $I_{n_{f} n_{i}}$ and $R_{i d}^{+*}$ for the singlet states of the helium atom.

\begin{tabular}{|c|c|c|c|c|c|c|c|}
\hline$n_{f} \backslash n_{i}$ & 1 & 2 & 3 & 4 & 5 & 6 & 7 \\
\hline \hline 1 & $\mathbf{0 . 9 2 9 5}$ & 0.0493 & 0.0136 & 0.0055 & 0.0028 & 0.0016 & 0.0010 \\
\hline 2 & 0.0446 & $\mathbf{. 5 3 4 6}$ & 0.0702 & 0.0231 & 0.0106 & 0.0059 & 0.0036 \\
\hline 3 & 0.0055 & 0.3993 & $\mathbf{. 1 6 6 8}$ & 0.0533 & 0.0237 & 0.0131 & 0.0079 \\
\hline 4 & 0.0018 & 0.0035 & 0.7319 & $\mathbf{. 0 0 1 9}$ & 0.0063 & 0.0051 & 0.0037 \\
\hline 5 & 0.0008 & 0.0017 & 0.0131 & 0.7761 & $\mathbf{. 0 5 9 4}$ & 0.0107 & 0.0029 \\
\hline 6 & 0.0005 & 0.0009 & 0.0001 & 0.1380 & 0.4997 & $\mathbf{. 1 4 3 0}$ & 0.0541 \\
\hline 7 & 0.0003 & 0.0005 & 0.0001 & 0.0003 & 0.3865 & 0.1606 & $\mathbf{. 1 1 2 9}$ \\
\hline 8 & 0.0002 & 0.0003 & 0.0001 & & 0.0103 & 0.5996 & 0.0025 \\
\hline 9 & 0.0001 & 0.0002 & 0.0001 & & & 0.0597 & 0.6106 \\
\hline 10 & 0.0001 & 0.0002 & 0.0001 & & & 0.0001 & 0.1969 \\
\hline 11 & 0.0001 & 0.0001 & & & & & 0.0035 \\
\hline 12 & & 0.0001 & & & & & 0.0000 \\
\hline \hline$R_{i d}^{+*}$ & & $\mathbf{. 0 5 2 3}$ & $\mathbf{. 0 9 1 7}$ & $\mathbf{. 0 8 9 3}$ & $\mathbf{. 0 4 5 2}$ & $\mathbf{. 0 3 7 8}$ & $\mathbf{. 0 7 9 0}$ \\
\hline
\end{tabular}

\section{INTRODUCTION}

The processes of two-electron photoionization and ionization with excitation have attracted the attention of theorists and experimentalists for a long time. A steady increase of activity has occurred during the last decade [1, 2, 3, 4, 5, 6, 7, 8]. The interest in these processes is motivated to a large extent by the desire to test our ability to calculate reliably the two-electron wave function and to understand the mechanisms of these processes that take place solely due to the interelectron interaction.

The simplest objects where the interelectron interaction can manifest itself are the twoelectron systems as the He atom and the helium-like ions. Therefore they represent the main targets of investigation. Recently a number of studies have been carried out of the two-electron photoionization cross sections of these systems, including the corresponding ratios of the two-electron and the single-electron cross sections, at high but nonrelativistic photon energies $\omega$ (see [9, 10, 11, 12] and references therein). 
TABLE II: The same as in Table I, but for the triplet states of the helium atom.

\begin{tabular}{|c|c|c|c|c|c|c|}
\hline$n_{f} \backslash n_{i}$ & 2 & 3 & 4 & 5 & 6 & 7 \\
\hline \hline 1 & .0338 & .0087 & .0034 & .0017 & .0009 & .0006 \\
\hline 2 &. $\mathbf{7 8 2 3}$ & .0591 & .0170 & .0073 & .0039 & .0023 \\
\hline 3 & .1733 &. $\mathbf{4 0 5 3}$ & .0902 & .0352 & .0177 & .0103 \\
\hline 4 & .0044 & .5231 &. $\mathbf{0 7 6 0}$ & .0419 & .0228 & .0137 \\
\hline 5 & .0014 & .0006 & .7595 & $\mathbf{. 0 0 5 9}$ & .0005 & .0016 \\
\hline 6 & .0006 & .0004 & .0526 & .6723 & $\mathbf{0 . 0 9 9 5}$ & .0247 \\
\hline 7 & .0003 & .0003 & .0001 & .2324 & .3434 & $\mathbf{. 1 4 7 0}$ \\
\hline 8 & .0002 & .0002 & & .0027 & .4812 & .0615 \\
\hline 9 & .0001 & .0001 & & & .0299 & .6144 \\
\hline 10 & .0001 & .0001 & & & & .1225 \\
\hline 11 & .0001 & .0001 & & & & .0012 \\
\hline 12 & & & & & & .0000 \\
\hline \hline$R_{i d}^{+*}$ & $\mathbf{. 0 3 5 0}$ & $\mathbf{. 0 7 2 8}$ & $\mathbf{. 1 2 4}$ & $\mathbf{. 0 9 4 2}$ & $\mathbf{. 0 4 7 9}$ & $\mathbf{. 0 5 6 2}$ \\
\hline
\end{tabular}

In high photon energy region the cross sections of two-electron processes can be expressed via the initial state wave functions. The initial states considered were the ground and excited states of He and the helium-like ions.

For high but nonrelativistic $\omega$, the dominating mechanisms of the two-electron ionization and ionization with excitation are twofold: shake-off and the initial state correlations. Both exhibit the same dependence on $\omega$ at high $\omega$. The contribution of the final-state interaction, where the second electron is excited or ionized due to a collision with the primary eliminated electron that absorbs the incoming photon, decreases faster with $\omega$ than shake-off. The quasifree mechanism [13] operates in the situation where both electrons are ionized and acquire almost equal energies. Therefore the quasi-free mechanism is not taken into consideration in the framework of the ionization with excitation or de-excitation.

If the initial state is excited, the elimination of one of the electrons can be accompanied not only by the excitation of the second one, but also by de-excitation. As far as we are aware, the de-excitation process almost completely escaped theoretical investigation. However, it 
TABLE III: Values of the ratios $I_{n_{f} n_{i}}$ and $R_{i d}^{+*}$ for the singlet states of the $\mathrm{Li}^{+}$ion.

\begin{tabular}{|c|c|c|c|c|c|c|c|}
\hline$n_{f} \backslash n_{i}$ & 1 & 2 & 3 & 4 & 5 & 6 & 7 \\
\hline \hline 1 & $\mathbf{. 9 7 1 6}$ & .0718 & .0210 & .0087 & .0044 & .0025 & .0016 \\
\hline 2 & .0157 &. $\mathbf{7 4 5 6}$ & .0516 & .0147 & .0064 & .0034 & .0020 \\
\hline 3 & .0023 & .1528 & $\mathbf{. 5 4 7 6}$ & .0835 & .0293 & .0141 & .0080 \\
\hline 4 & .0008 & .0102 & .3561 &. $\mathbf{3 0 0 0}$ & .0813 & .0347 & .0183 \\
\hline 5 & .0004 & .0032 & .0082 & .5824 & $\mathbf{. 1 0 5 3}$ & .0523 & .0276 \\
\hline 6 & .0002 & .0015 & .0029 & .0009 & .7627 & $\mathbf{. 0 1 0 0}$ & .0177 \\
\hline 7 & .0001 & .0008 & .0014 & .0013 & .0052 & .8381 &. $\mathbf{0 1 0 7}$ \\
\hline 8 & .0001 & .0005 & .0008 & .0008 & .0003 & .0417 & .7807 \\
\hline 9 & .0001 & .0003 & .0005 & .0005 & .0003 & .0000 & .1314 \\
\hline 10 & .0000 & .0002 & .0003 & .0003 & .0002 & .0001 & .0000 \\
\hline 11 & .0000 & .0002 & .0002 & .0002 & .0002 & .0001 & .0000 \\
\hline 12 & .0000 & .0001 & .0002 & .0002 & .0001 & .0001 & .0000 \\
\hline \hline$R_{i d}^{+*}$ & & $\mathbf{. 0 7 8 4}$ & $\mathbf{. 0 7 9 0}$ & $\mathbf{. 1 2 0}$ & $\mathbf{. 1 3 9}$ & $\mathbf{. 1 2 0}$ & $\mathbf{. 0 8 1 5}$ \\
\hline
\end{tabular}

is expressed by integrals similar but not identical to those entering the expressions of the two-electron ionization and ionization with excitation cross sections [9, 10, 11, 14]. The aim of this paper is to study the photoionization accompanied by de-excitation. In principle, this process can be separated experimentally from the other two-electron processes, i.e., the double ionization and ionization with excitation, if the photoelectron's energy for the given incoming photon frequency $\omega$ is detected.

Here we obtain the initial state wave function using the correlation function hyperspherical harmonic method (CFHHM). The local accuracy of this wave function was previously studied in Ref.[15] for the ground and the $2^{1} S$ state of the helium atom, where it was shown that the local deviation of CFHHM wave function from the exact value is extremely small. Very accurate nonvariational CFHHM wave functions of the He atom and the helium-like ions in their ground and several lowest excited ${ }^{1} S$ and ${ }^{3} S$ states [16, 17] were utilized therefore to calculate the cross sections of the processes of interest. We calculated the high-energy photoionization cross sections that can be expressed solely via the initial state two-electron 
TABLE IV: The same as in Table III, but for the triplet states of the $\mathrm{Li}^{+}$ion.

\begin{tabular}{|c|c|c|c|c|c|c|}
\hline$n_{f} \backslash n_{i}$ & 2 & 3 & 4 & 5 & 6 & 7 \\
\hline \hline 1 & .0559 & .0158 & .0064 & .0032 & .0018 & .0011 \\
\hline 2 &. $\mathbf{8 7 6 2}$ & .0316 & .0079 & .0032 & .0017 & .0010 \\
\hline 3 & .0574 &. $\mathbf{7 2 8 3}$ & .0771 & .0241 & .0109 & .0060 \\
\hline 4 & .0044 & .2085 &. $\mathbf{4 7 8 8}$ & .0982 & .0375 & .0188 \\
\hline 5 & .0013 & .0070 & .4194 &. $\mathbf{2 3 4 0}$ & .0836 & .0391 \\
\hline 6 & .0006 & .0022 & .0031 & .6321 &. $\mathbf{0 6 4 9}$ & .0467 \\
\hline 7 & .0003 & .0010 & .0016 & .0003 & .7789 &. $\mathbf{0 0 1 1}$ \\
\hline 8 & .0002 & .0005 & .0008 & .0006 & .0179 & .8094 \\
\hline 9 & .0001 & .0003 & .0005 & .0004 & .0001 & .0747 \\
\hline 10 & .0001 & .0002 & .0003 & .0003 & .0001 & .0000 \\
\hline 11 & .0001 & .0002 & .0002 & .0002 & .0002 & .0001 \\
\hline 12 & .0000 & .0001 & .0001 & .0001 & .0001 & .0000 \\
\hline \hline$R_{i d}^{+*}$ & $\mathbf{. 0 5 9 4}$ & $\mathbf{. 0 4 9 9}$ & $\mathbf{. 1 0 1}$ & $\mathbf{. 1 4 8}$ & $\mathbf{. 1 5 7}$ & $\mathbf{. 1 2 7}$ \\
\hline
\end{tabular}

wave functions $\Psi_{i}\left(\mathbf{r}_{1}, \mathbf{r}_{2}\right)$.

In this work we will use these nonvariational wave functions for the He atom and the helium-like ions in several lowest excited ${ }^{1} S$ and ${ }^{3} S$ states to calculate the high-energy limits of the cross sections of photoionization with de-excitation. The results of these limits will be compared with the cross sections of the photoionization with excitation and with the single-electron photoionization cross sections. To study the theoretically interesting nuclear charge dependences of considered values, we investigate also the $\mathrm{Li}^{+}$and $\mathrm{B}^{3+}$ ions.

Unfortunately, till now in absolute majority of experiments only the double- and singlecharged ions are counted. Therefore, the excitation and de-excitation processes remained not detected but included into the yield of single charge ions. 
TABLE V: Values of the ratios $I_{n_{f} n_{i}}$ and $R_{i d}^{+*}$ for the singlet states of the $\mathrm{B}^{3+}$ ion.

\begin{tabular}{|c|c|c|c|c|c|c|c|}
\hline$n_{f} \backslash n_{i}$ & 1 & 2 & 3 & 4 & 5 & 6 & 7 \\
\hline \hline 1 &. $\mathbf{9 9 0 5}$ & .0892 & .0270 & .0114 & .0058 & .0034 & .0020 \\
\hline 2 & .0047 &. $\mathbf{8 4 8 5}$ & .0235 & .0057 & .0023 & .0012 & .0007 \\
\hline 3 & .0008 & .0448 &. $\mathbf{8 1 5 6}$ & .0495 & .0138 & .0060 & .0031 \\
\hline 4 & .0003 & .0054 & .1098 & $\mathbf{. 7 0 5 3}$ & .0725 & .0228 & .0102 \\
\hline 5 & .0001 & .0018 & .0094 & .2009 &. $\mathbf{5 6 6 3}$ & .0877 & .0300 \\
\hline 6 & .0001 & .0009 & .0030 & .0116 & .3131 & $\mathbf{. 4 1 9 7}$ & .0900 \\
\hline 7 & .0000 & .0005 & .0014 & .0036 & .0112 & .4376 &. $\mathbf{2 7 4 6}$ \\
\hline 8 & .0000 & .0003 & .0008 & .0017 & .0037 & .0080 & .5493 \\
\hline 9 & .0000 & .0002 & .0005 & .0010 & .0018 & .0030 & .0036 \\
\hline 10 & .0000 & .0001 & .0003 & .0006 & .0009 & .0019 & .0015 \\
\hline 11 & .0000 & .0001 & .0002 & .0004 & .0007 & .0006 & .0036 \\
\hline 12 & .0000 & .0001 & .0002 & .0003 & .0004 & .0007 & .0000 \\
\hline \hline$R_{i d}^{+*}$ & &. $\mathbf{0 9 8 7}$ &. $\mathbf{0 5 3 6}$ &. $\mathbf{0 7 1 8}$ & $\mathbf{. 1 0 5}$ & $\mathbf{. 1 3 8}$ & $\mathbf{. 1 5 8}$ \\
\hline
\end{tabular}

\section{MAIN FORMULAS}

We start from the formula for the two-electron photoionization cross section at asymptotically high $\omega$ obtained in 9] that has some recent derivations [10, 11]. The expression for the cross section $\sigma^{+*}(\omega)$ of the ionization with excitation, in this $\omega$ region, for the He atom and the helium-like ions in their excited states, is accordingly as follows:

$$
\sigma_{i}^{+*}(\omega)=\frac{32 Z^{2} \sqrt{2} \pi^{2}}{3 c \omega^{7 / 2}} \sum_{n_{f}} I_{n_{f} n_{i}},
$$

where $I_{n_{f} n_{i}}$ is the overlap integral defined as

$$
I_{n_{f} n_{i}}=4 \pi\left|\int_{0}^{\infty} \Psi_{i}(0, r) R_{n_{f} 0}(r) r^{2} d r\right|^{2} .
$$

Here $Z$ is the nuclear charge, $i\left(n_{i}\right)$ denotes the initial state, $R_{n_{f} 0}(r)$ are the hydrogenic single-electron radial wave functions with the principal quantum number $n_{f}$ and angular momentum zero. We note that the excitations of states with nonzero angular momenta $l$ decrease faster than $\omega^{-7 / 2}$ : namely as $\omega^{(-7 / 2+l)}$. 
TABLE VI: The same as in Table V, but for the triplet states of the $\mathrm{B}^{3+}$ ion.

\begin{tabular}{|c|c|c|c|c|c|c|}
\hline$n_{f} \backslash n_{i}$ & 2 & 3 & 4 & 5 & 6 & 7 \\
\hline \hline 1 & .0764 & .0228 & .0095 & .0048 & .0028 & .0017 \\
\hline 2 &. $\mathbf{9 0 2 5}$ & .0122 & .0027 & .0010 & .0005 & .0003 \\
\hline 3 & 0.0162 &. $\mathbf{8 9 1 4}$ & .0359 & .0091 & .0038 & .0020 \\
\hline 4 & .0019 & .0613 &. $\mathbf{8 0 0 4}$ & .0622 & .0179 & .0078 \\
\hline 5 & .0006 & .0054 & .1337 & $\mathbf{. 6 7 2 2}$ & .0840 & .0272 \\
\hline 6 & .0003 & .0017 & .0084 & .2306 & $\mathbf{. 5 2 6 5}$ & .0964 \\
\hline 7 & .0002 & .0008 & .0025 & .0095 & .3460 &. $\mathbf{3 7 8 3}$ \\
\hline 8 & .0001 & .0004 & .0012 & .0030 & .0082 & .4717 \\
\hline 9 & .0001 & .0003 & .0006 & .0014 & .0028 & .0045 \\
\hline 10 & .0000 & .0002 & .0004 & .0008 & .0014 & .0023 \\
\hline 11 & .0000 & .0001 & .0003 & .0005 & .0008 & .0013 \\
\hline 12 & .0000 & .0001 & .0002 & .0003 & .0005 & .0005 \\
\hline \hline$R_{i d}^{+*}$ & $\mathbf{. 0 8 2 9}$ & $\mathbf{. 0 3 6 4}$ &. $\mathbf{0 5 0 7}$ & $\mathbf{. 0 8 3 9}$ & $\mathbf{. 1 2 3}$ & $\mathbf{. 1 5 7}$ \\
\hline
\end{tabular}

The photoionization cross section $\sigma^{+}(\omega)$ of the inner electron, without alteration of the state of the outer one, is given by the following expression

$$
\sigma_{i}^{+}(\omega)=\frac{128 Z^{2} \sqrt{2} \pi^{3}}{3 c \omega^{7 / 2}}\left|\int_{0}^{\infty} \Psi_{i}(0, r) R_{n_{i} 0}(r) r^{2} d r\right|^{2} .
$$

The cross section $\sigma_{(d)}^{+*}$ of the photoionization with de-excitation is calculated using the expressions (1) and (2), where the summations over $n_{f}$ include different than for ionization with excitation values. As was mentioned above, we classify the initial state by its principal quantum number $n_{i}$. The ground state can be considered approximately as a state with $n_{i}=1$. The next state is $n_{i}=2$ and so on: the higher the excitation, the more precise becomes the assignment of a given state to an integer principal quantum number. With increasing excitation principal quantum number $n_{i}$, the wave function approaches a symmetrized product of two pure Coulomb wave functions. One is an $1 s$ electron function in the nuclear field with charge $Z$, and the other is a function in the $(Z-1)$ field. Thus $\sigma^{+*}(\omega)$ includes the summation over $n_{f}>n_{i}$ while $\sigma_{i(d)}^{+*}(\omega)$ includes the summation over $n_{f}<n_{i}$. 
The ratio $R_{i d}^{+*}$ of the cross section $\sigma_{i(d)}^{+*}(\omega)$ and the sum of $\sigma_{i}^{+}(\omega)$ and $\sigma_{i}^{+*}(\omega)$ is given by the expression:

$$
R_{i d}^{+*} \equiv \frac{\sigma_{i(d)}^{+*}(\omega)}{\sigma_{i}^{+}(\omega)+\sigma_{i}^{+*}(\omega)}=\frac{\sum_{n_{f}<n_{i}} I_{n_{f} n_{i}}}{\sum_{n_{f} \geq n_{i}} I_{n_{f} n_{i}}} .
$$

It is seen from Eq.(2) that $\sigma_{i}^{+*}$ and $\sigma_{i(d)}^{+*}$ probe different parts of initial state wave function $\Psi_{i}(0, r)$.

\section{RESULTS OF CALCULATIONS}

The Tables present the results of our calculations for the singlet and triplet states of the He atom (Tables I and II), and the helium-like ions $\mathrm{Li}^{+}$(Tables II and IV) and $\mathrm{B}^{3+}$ (Tables $\mathrm{V}$ and VI).

Most results can be obtained using a small hyperspherical harmonic ( $\mathrm{HH})$ basis with $K_{m}=48$, for some ground states even with $K_{m}=40$, where $K_{m}$ is the maximum global angular momentum used in the $\mathrm{HH}$ expansion. The main problem is the calculation of the overlap integrals (2) between the accurate CFHHM wave function and the hydrogenic single-electron ones of the highest orders. The very precise calculations of the CFHHM wave function $\Psi_{i}(0, r)$ at one electron-nucleus zero-distance for large values of $r$ are required. At that, the precision of the CFHHM wave function, including its values for the large $r$, increases slowly with increasing $K_{m}$, while the calculation time increases considerably with $K_{m}$ growth. We used here the HH basis with $K_{m}=100$ for the excited $2 S-7 S$ both singlet and triplet states of He atom and the one with $K_{m}=64$ for the other ions in the excited states, which make the calculation quite difficult. This gives data with accuracy well above obtainable experimentally in foreseeable future.

In the Tables we presented only several the first terms $\left(n_{f} \leq 12\right)$ of the set of integrals (2). However, we have used 200 single-particle states to calculate the cross-section ratios, so that the error of summation over states at least is less than the error of the data presented in the Tables.

\section{DISCUSSION AND CONCLUSIONS}

In all cases the probability of the initially excited electron to remain on the same level $\left(n_{f}=n_{i}\right)$ decreases with $n_{i}$. In principle, this is quite natural since the higher the $n_{i}$, the 
easier it is to alter the electron state in the course of the rapid elimination of the $1 S$ electron. This decrease is monotonic for pure hydrogenic functions; in the lowest order in interelectron interaction, that result can be demonstrated analytically [18]. For the He atom the decrease is non-monotonic and the overlap integral Eq. (2) reaches its minimum value at $n_{i}=n_{f}=4$ for the singlet states and at $n_{i}=n_{f}=5$ for the triplet states. This is the consequence of the strong deviation of $\Psi_{i}(0, r)$ from its simplest approximation. A trace of the helium-like behavior can be found in the minimum of $I_{n_{f} n_{i}}$ at $n_{i}=n_{f}=7$ for singlet states in $\mathrm{Li}^{+}$.

The $\mathrm{B}^{3+}$ ion is already purely hydrogenlike, the role of its interelectron interaction being relatively smaller than in He. This brings about the decrease of $I_{n_{f} n_{i}}$ for $n_{f}=n_{i}$ with increasing $n_{i}$. As a result, the probability for the initially excited electron to remain on the same level after the inner $1 s$ electron is photoionized increases. Indeed, $I_{77}$ for $\mathrm{B}^{3+}$ is about 30 times bigger than for $\mathrm{Li}^{+}$. The corresponding ratio for the triplet $I_{77}$ values is even larger by an additional factor of 15. As seen from the Tables, the most probable process is the ionization with excitation to the next one or two levels. The ratio $R_{i d}^{+*}$ varies relatively little, from 0.035 to 0.158 . It has quite a complex form, with at least two minima for the He atom and at least one minimum for the singlet and triplet states of $\mathrm{B}^{3+}$.

Let us have in mind, that the process of photoionization with excitation can be relatively easily distinguished from other two-electron processes, particularly from the two-electron ionization. This can be achieved simply by detecting photoelectrons with energies larger than the energy of the incoming photon. The ratio $R_{i d}^{+*}$ of the cross section of the ionization with de-excitation and the cross section of the ionization with excitation presents a rather complex function of the initial state and is completely determined by its wave function. Observation of this process could serve as an additional to studies of ionization with excitation, verification of the quality of the wave functions employed in describing the initial state of the target atoms or ions.

Note, that a target consisting of excited atoms can be produced by initial illumination of a He gas volume by e.g. laser light. The $p$-excited states then radiatively decay into excited $s$-states.

Targets of triplet excited states can be produced by colliding at small angles of $\alpha$-particles beem with a magnetized, i.e. occupied by having electrons with the same spin orientation metallic surface.

The development of experimental technique and growth of intensity of available high pho- 
ton beams will lead without doubt to the experiments, in which for a given photon frequency the outgoing photoelectron's energy will be detected accurately enough. It will permit to study ionization with excitation and de-excitation. A good object for de-excitation studies would be the $2^{3} S$ state of helium the lifetime of which is about eight minutes. Experimental detection of ionization with de-excitation is, of course, an experimental challenge and we do believe that it will attract experimentalists very soon.

\section{Acknowledgments}

We acknowledge the financial support of the Binational Science foundation under the grant 2002064 and the Israeli Science foundation under the grant 174/03.

[1] T. A. Carlson, Phys. Rev. 156, 142 (1967).

[2] F. W. Byron and C. J. Joachain, Phys. Rev. 164, 1 (1967).

[3] T. Aberg, Phys. Rev. A 2, 1736 (1970).

[4] A. Dalgarno and H. Sadeghpour, Phys. Rev. A 46, 3591 (1992).

[5] J. H. McGuire, N. Berrah, R. J. Bartlett, J. A. R. Samson, J. A. Tanis, C. L. Cocke, and A. S. Schlachter, J. Phys. B 28, 913 (1995).

[6] A. S. Kheifets and I. Bray, Phys. Rev. A 54, R995 (1996).

[7] J. H. McGuire, Electron Correlation Dynamics in Atomic Collisions, Cambridge University Press, 1997.

[8] J. Ullrich, R. Moshammer, R. O. Jagutzki, V. Mergel, H. Schmidt-Boking and L. Spielberger, J. Phys. B 30, 2917 (1997).

[9] R. Krivec, M. Ya. Amusia, and V. B. Mandelzweig, Phys. Rev. A 62, 064701 (2000).

[10] R. Krivec, M. Ya. Amusia, and V. B. Mandelzweig, Phys. Rev. A 63, 052708 (2001).

[11] R. Krivec, M. Ya. Amusia, and V. B. Mandelzweig, Phys. Rev. A 67(6), 62720-7 (2003).

[12] E. G. Drukarev, M. Ya. Amusia, R. Krivec and V. B. Mandelzweig, Few Body Systems 35, 3 (2004).

[13] M. Ya. Amusia, E. G. Drukarev, V. G. Gorshkov, and M. P. Kazachkov, J. Phys. B 8, 1248 (1975). 
[14] M. Ya. Amusia, Atomic Photoeffect, Plenum Press, New York and London, 1990.

[15] M. I. Haftel and V. B. Mandelzweig, Phys. Rev. A 42, 6324 (1990).

[16] M. I. Haftel, R. Krivec, and V. B. Mandelzweig, J. Comp. Phys. 123, 149 (1996).

[17] R. Krivec, V. B. Mandelzweig, and K. Varga, Phys. Rev. A 61, 062503 (2000).

[18] M. Ya. Amusia, A. S. Baltenkov, 1993, unpublished. 